\title{
A LINEAR MAPPING BETWEEN FUNCTION SPACES
}

\section{E. MICHAEL ${ }^{1}$}

1. Introduction. Suppose that $X$ and $Y$ are compact, and let $C(X)$ and $C(Y)$ denote the Banach spaces of real-valued continuous functions on $X$ and $Y$. Let $p$ be a function from $X$ onto $Y$. If $p$ admits a continuous cross section $q: Y \rightarrow X$ (i.e., $q(y) \in p^{-1}(y)$ for every $y$ in $Y$ ), then the adjoint map $u: C(X) \rightarrow C(Y)$ of $q$, defined by

$$
[u(f)](y)=f(q(y)),
$$

is a continuous linear transformation from $C(X)$ onto $C(Y)$, satisfying the following condition for every $f \in C(X)$ and $y \in Y$ :

$$
\inf \left(f\left[p^{-1}(y)\right]\right) \leqq[u(f)](y) \leqq \sup \left(f\left[p^{-1}(y)\right]\right) .^{2}
$$

The purpose of this note is to show that, even if $p$ does not admit a cross section, such a mapping $u$ always exists, provided $p$ is open and $X$ and $Y$ are metrizable. That answers a question asked by Fred B. Wright.

THEOREM 1.1. Let $X$ and $Y$ be compact metric spaces, and $p$ an open map from $X$ onto $Y$. Then there exists a continuous linear mapping $u$ from $C(X)$ onto $C(Y)$, satisfying (1) for every $f \in C(X)$ and $y \in Y$.

The requirement that $p$ be open cannot be dropped, as the following example shows.

EXAMPLE 1.2. Let $X$ be the interval [0,3], $Y$ the interval obtained from $X$ by identifying all points in $[1,2]$, and $p: X \rightarrow Y$ the natural projection. If $f \in C(X)$ is 0 on $[0,1]$ and 1 on $[2,3]$, then there exists no possible $u(f) \in C(Y)$ satisfying (1).

I don't know whether metrizability of $X$ and $Y$ in Theorem 1.1 can be weakened to Hausdorff, but would like to conjecture that it can't.

2. Proof of Theorem 1.1. Our proof is based on the following lemma, which was essentially proved in [3] or [4].

Presented to the Society, April 8, 1961 under the title $A$ linear transformation between function spaces; received by the editors February 2, 1963.

1 Supported by a National Science Foundation contract.

2 Note that any linear $u: C(X) \rightarrow C(Y)$ which satisfies (1) is automatically continuous (with norm 1) and onto (since, for any $g \in C(Y), u(g \circ p)$ must be $g$ ). 
Lemma 2.1. Let $Y$ be a paracompact space, $F$ a complete metrizable locally convex space, and $\phi: Y \rightarrow 2^{F}$ lower semi-continuous. ${ }^{3}$ Then there exists a continuous $h: Y \rightarrow F$ such that

$$
h(y) \in(\operatorname{conv}(\phi(y)))^{-}
$$

for every $y \in Y$.

Proof. If every $\phi(y)$ is closed and convex, this is asserted in [3, Theorem 3.2] or [4, Theorem 1]. ${ }^{4}$ In general, define $\psi: Y \rightarrow 2^{F}$ by $\psi(y)=(\operatorname{conv}(\phi(y)))$. Then $\psi$ is also lower semi-continuous [3, Propositions 2.3 and 2.6], and we may apply the above selection theorem to $\psi$. That completes the proof of the lemma.

Let us now prove Theorem 1.1. Denote the Banach space $C(X)$ by $E$, let $E^{*}$ be its dual space, and let $S^{*}$ be the unit sphere of $E^{*}$. Then $S^{*}$ is a compact convex subset of $E^{*}$, equipped with the topology $\sigma$ of pointwise convergence on $E$. By identifying each $x \in X$ with the element $\tilde{x} \in S^{*}$ defined by $\tilde{x}(f)=f(x)$, we may consider $X$ as a subset of $\left(S^{*}, \sigma\right)$.

Since $X$ is compact metric, $E$ has a countable dense subset $A[2$, (7.4.4)]. Since $S^{*}$ is equicontinuous, $\sigma$ coincides on $S^{*}$ with the topology $\sigma^{\prime}$ of pointwise convergence on $A$ [1, p. 29, Proposition 3]. Now $\left(E^{*}, \sigma^{\prime}\right)$ is first-countable, and hence metrizable. Denoting the completion of $\left(E^{*}, \sigma^{\prime}\right)$ by $F$, we therefore see that $F$ is a complete metrizable locally convex space; moreover, $F$ contains the compact (and hence closed) convex subset $\left(S^{*}, \sigma\right)=\left(S^{*}, \sigma^{\prime}\right)$, and this set contains $X$.

Define $\phi: Y \rightarrow 2^{F}$ by $\phi(y)=p^{-1}(y)$. Since $p$ is open, $\phi$ is lower semicontinuous. Applying Lemma 2.1, we obtain a continuous $h: Y \rightarrow F$ such that

$$
h(y) \subset(\operatorname{conv}(\phi(y)))^{-}
$$

for every $y \in Y$. Now $h$ is not a cross section for $p$, since its range is not even contained in $X$. However, $h(y) \in S^{*} \subset E^{*}$ for all $y \in Y$, so we may still take $u$ to be the adjoint of $h$, defined by

$$
[u(f)](y)=f(h(y))
$$

for $f \in C(X)$ and $y \in Y$. This $u$ is clearly linear and satisfies (1), and hence (as noted in footnote 2 ) is continuous and onto. That completes the proof.

${ }^{3}$ I.e., $\{y \in Y \mid \phi(y) \cap V \neq \varnothing\}$ is open in $Y$ for every open $V \subset F$.

4 The results in [3] and [4] are actually stated only for Banach spaces $F$, but, as remarked on page 364 of [3], the proof in [3] remains valid (without change) under our weaker assumptions. 
3. Generalizations. Theorem 1.1 can be generalized in two directions. In the first place, $C(X)$ and $C(Y)$ can be replaced by $C(X, B)$ and $C(Y, B)$, where $B$ is any Banach space, provided (1) is changed to

$$
[u(f)](y) \in\left(\operatorname{conv}\left(f\left[p^{-1}(y)\right]\right)\right)^{-} .
$$

Moreover, if $B$ is a complex Banach space (in particular, the complex numbers), then $u$ may be chosen complex-linear. For separable $B$, this result is proved just as is Theorem 1.1, except that the dual of $C(X)$ is replaced by the space of bounded linear transformations from $C(X, B)$ to $B$ (real or complex, as the case may be). If $B$ is not separable, the proof becomes more complicated, and will be given in [5].

The other direction for generalizing Theorem 1.1 is to require only that $X$ and $Y$ be metric spaces (not necessarily compact) and that each $p^{-1}(y)$ is complete. In this case, $C(X)$ and $C(Y)$ denote the spaces of all continuous real-valued functions on $X$ and $Y$, with the compact-open topology. (As a matter of fact, this result also remains true with $C(X)$ and $C(Y)$ replaced by $C(X, B)$ and $C(Y, B)$, as above.) The proof will be given in [5].

\section{REFERENCES}

1. N. Bourbaki, Topologie générale, Chapter X, Hermann, Paris, 1949.

2. J. Dieudonne, Foundations of modern analysis, Academic Press, New York, 1960.

3. E. Michael, Continuous selections. I, Ann. of Math. (2) 63 (1956), 361-382.

4. - Selected selection theorems, Amer. Math Monthly 63 (1956), 233-238.

5. - Three mapping theorems, Proc. Amer. Math. Soc. 15 (1964), 410-415.

UNIVERSITY OF WASHINGTON 